\title{
Terrain Model Acquisition By Mobile Robot Teams and $n$-Connectivity
}

\author{
Nageswara S. V. Rao ${ }^{1}$ \\ Center for Engineering Science Advanced Research, Computer Science and \\ Mathematics Division, Oak Ridge National Laboratory, Oak Ridge, TN \\ 37831-6355; email:raons@ornl.gov.
}

\begin{abstract}
The connectivity of the configuration space has been a valuable concept in the motion planning for single robots in both known and unknown terrains. We show here that $n$-connectivity plays a similar role for mobile robot teams in providing algorithms for terrain model acquisition in unknown terrains. A bound on the connectivity degree of the free-space, reflected in that of a navigation course, provides us an estimate of the size of a robot team that is effective for the terrain. We consider an unknown planar polygonal terrain. The robots are point-sized and equipped with visual sensors which acquire all visible parts of the terrain by scan operations executed from their locations. The performance is measured by the number of sensor (scan) operations which are assumed to be the most time-consuming of all the robot operations. We show that the Voronoi diagrams and trapezoidal decomposition methods yield solutions for efficient terrain model acquisition by a 2- and 3-robot team using visual sensors.
\end{abstract}

\section{Introduction}

The terrain model acquisition problem deals with robots autonomously acquiring a complete model of an unknown terrain by systematically visiting portions of it. There are several motivations for this problem. Once the terrain model is completely acquired, the navigation algorithms of known terrains can be employed for path planning with two potential advantages. First, the sensors may be switched off (at least in theory) in future navigation, saving the time and resources involved in the acquisition and processing of sensor data. Second, navigation paths with the shortest distance between the start and goal positions may be computed using the terrain model. In applications involving mobile robots in indoor environments for repetitive operations, typically a human operator is in charge of model building, which is tedious and time-consuming. Robots capable of autonomous terrain model acquisition (even in only small parts of the terrain) can assist in the model building process.

The terrain model acquisition problem (TMAP) for polyhedral or polygonal terrains has been solved using a single robot in $[14,13,18,12]$ for the case of a discrete vision sensor. TMAP for a robot equipped with a continuous vision sensor has been solved in [9,12]. TMAP for team of robots has been recently addressed by a number of investigators. Ishioka et al. [6] described 
a cooperative map generation by heterogeneous autonomous mobile robots (also see Dudek et al. [3]). A cooperative recognition system for the environment using multiple robots has been developed by Ishiwata et al. [7]. Rao et al $[17,16]$ addressed this problem in the formulation of convergent algorithms using visibility graphs. In particular, if all obstacles are convex, the sensing time was shown to be essentially reduced by a factor of $1 / n$ for $n=2,3,4$. The disadvantage of the visibility graph method is that robots must be capable of navigating very close to the obstacles.

In this paper, we show that the navigational structures that keep the robots away from the obstacle boundaries can also be utilized by an $n$-robot team for $n=2,3$. There are other problems in unknown terrains studied for mobile robot teams. The study by Harinarayan and Lumelsky [5] indicates that the simultaneous navigation of two robots cannot be solved if no "cooperation" is present between them. Note that our overall objective is different from theirs in two ways, namely: (a) we are interested in terrain model acquisition, and (b) we wish to explore the cooperation mechanisms so that the objective can be achieved more effectively by a team of robots instead of a single robot.

There are obvious limiting cases in deploying mobile robot teams for the terrain model acquisition. In "very bad" cases, e. g. the entire team of robots is initially located at one end of a "long narrow polygonal corridor", there may not be any advantage in employing a team. Robots are forced to form a single cluster, thereby obviating any advantages of a team. However, if the terrain has "branches", a team is likely to acquire the terrain faster than one robot. In this paper, we discuss general results that indicate the conditions under which a robot team is more effective from an algorithmic perspective. We show that for 2- and 3-robot teams the navigation structures based on the Voronoi diagram and trapezoidal decomposition methods reduce the sensing time, while keeping the robots wary from obstacle boundaries. More generally, we show that the $n$-connectivity of the navigation course, which reflects the connectivity structure of the configuration space, enables an $n$-robot team to reduce the sensor time by a factor of $1 / n$ compared to a single robot. We show that Voronoi diagram and dual graph based on trapezoidal decomposition are 2-connected if the obstacles are all convex. For 3-robot team, these navigational courses offer good solutions although they do not reduce the sensor time by $1 / 3$. For general polygonal terrains, the reductions may not be as dramatic, but a reduction in sensor time is achieved depending on the connectivity structure of the configuration space.

Preliminaries are described in Section 2. Algorithmic methods for terrain model acquisition by a single robot are discussed in Section 3 . The concepts of $n$-connectivity and its implications on robot team exploration are discussed in Section 4. The cases of convex and non-convex polygonal obstacles are discussed in Section 5 and 6, respectively. 


\section{Preliminaries}

We consider a finite two-dimensional terrain cluttered by a finite and nonintersecting set of polygonal obstacles. An obstacle vertex is convex if the angle included inside the obstacle by the edges that meet at this vertex is less than 180 degrees; otherwise the obstacle vertex is concave. Two points $p$ and $q$ in plane are visible to each other if the line segment joining $p$ and $q$, denoted by $\overline{p q}$, lies entirely outside the interior of all obstacle polygons.

The robot, denoted by $R$, is point-sized and equipped with a vision sensor. A discrete vision sensor is characterized by a scan operation: a scan operation performed from a position (point) $p$ returns the visibility polygon of $p$ that consists of all points in the terrain visible to $p$ (Fig. 1). We assume that the most time consuming-part of the robot operation is the scan operation. In vision-based robots, each scan may take several minutes including the time required to acquire and process the sensory data. The total sensing time is given by the number of scan operations performed by the robot(s) in sequence. The robots communicate with each other via a wireless network.

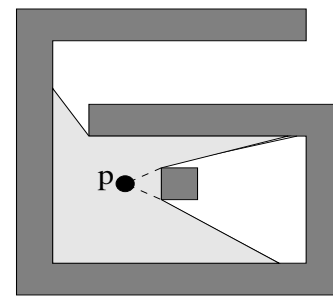

visibility polygon

obstacle polygon

Fig. 1. Visibility polygon from location $p$.

\section{Navigational Courses For TMAP}

An algorithmic paradigm to solve TMAP has been proposed by Rao [10]. A finite graph called the navigation course $\xi$, is used as an underlying structure. Initially navigation course is not known, but it is incrementally constructed from the sensor operations by the robot systematically visiting all vertices of the navigation course.

In order that a graph exploration algorithm terminates, the navigation course must contain a finite number of edges and vertices, i.e., must satisfy finiteness property. It must satisfy the terrain-visibility property which requires that every point in the free-space is visible from some vertex of the navigation course. It must also satisfy the connectivity, more precisely 1-connectivity, property which requires that every pair of vertices be connected by a graph path on the navigation course. We require that adjacency list of a $\xi$-vertex can be constructed from the information of a single scan; this property is called the local-constructibility. For a navigation course $\xi$ that 
satisfies the properties of finiteness, connectivity, terrain-visibility and localconstructibility, any graph search algorithm (e.g. depth-first search) can be employed to solve the navigation and the terrain model acquisition problems.

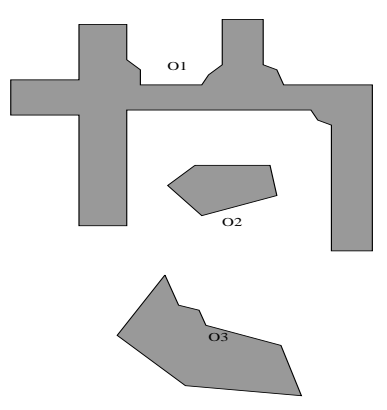

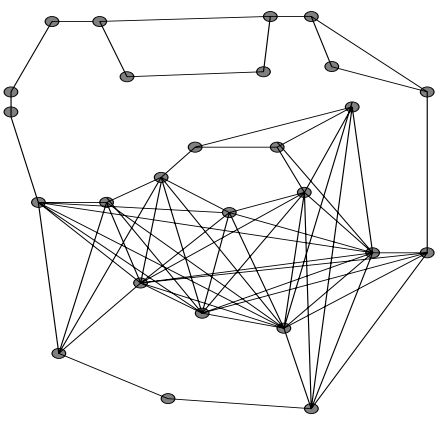

Fig. 2. Restricted visibility graph.

We now discuss three examples of the navigation course. The restricted visibility graph, $R V G=(V, E)$ is defined as follows [13]: (a) $V$ is the set of all convex obstacle vertices, (b) an edge $\left(v_{1}, v_{2}\right)$, for $v_{1}, v_{2} \in V$ represents the fact that the line joining $v_{1}$ and $v_{2}$ either corresponds to an obstacle edge or does not intersect any obstacle polygon. See Fig. 2 for an example of $R V G$.
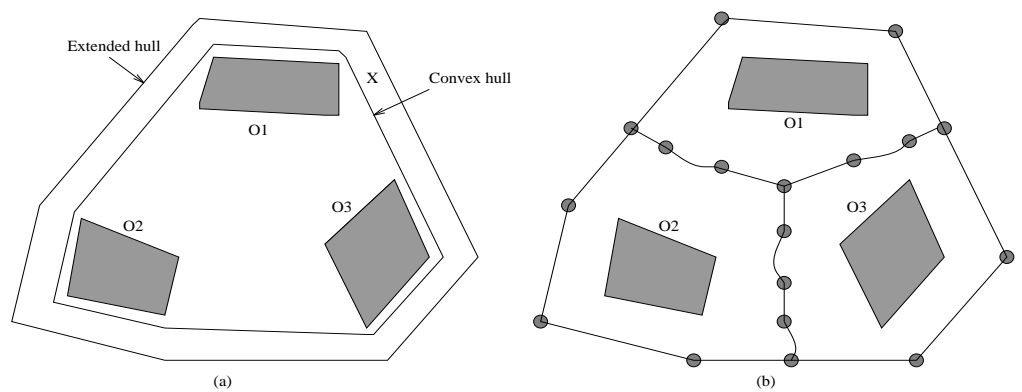

Fig. 3. Voronoi Diagram of Terrain

The second structure $V D$ is based on the Voronoi diagram, and can be described as follows [18]. Consider terrain $O$ of Fig. 3. The convex hull $C H$ of $O$ is the minimal polygonal region that encloses all obstacle polygons. The extended hull $E H$ is the convex polygonal region obtained by pushing out the edges of $C H$ by a distance $x$. The Voronoi diagram of the terrain is the locus of points that are closest to at least two points on the obstacle boundary. The Voronoi diagram consists of straight line segments and second 
order curve segments. The $V D$ is obtained by taking the union of the Voronoi diagram contained in $E H$, and the boundary of $E H$ as in Fig. 3(b).

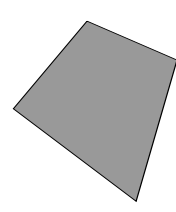

(a) Terrain

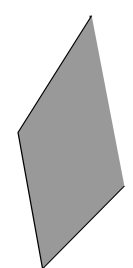

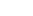

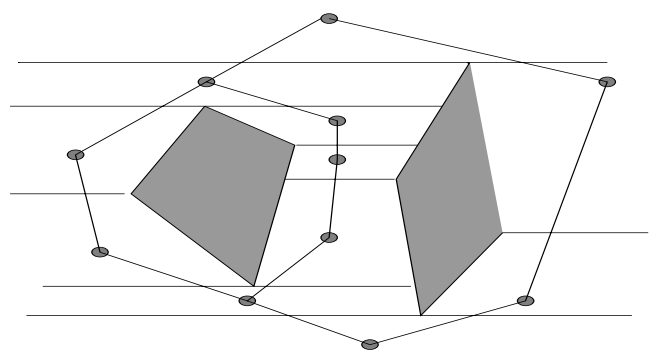

(b) Dual Graph of Terrain

Fig. 4. Dual Graph of Terrain

There are many other ways of generating navigational courses, based on dual graphs corresponding to decompositions such as trapezoidal decomposition, triangulation, etc., of free space [12] (also see [11]). Consider the terrain of Fig. 4. We decompose free-space into trapezoids by sweeping a horizontal line. When this line reaches a vertex, we extend (at most two and at least one) line segments from this vertex into free-space until obstacle boundary is reached or to infinity. The free-space is then decomposed into trapezoids by these line segments. Then the dual graph DG is obtained by denoting each trapezoid by a node and joining two nodes by an edge if and only if the corresponding trapezoids share a boundary edge.

The terrain model acquisition problem was first formulated and solved for two and three dimensional terrains by Rao et al [14] using the visibility graph. When the robot is circular in shape, Rao and Iyengar [13] proposed a navigation course based on the visibility graph. For a robot of polygonal shape with an ability to translate in any direction, Foux et al. [1993] proposed a navigation algorithm. See [15] for a survey (also see [2]). The terrain model acquisition method based on the extended visibility graph method was proposed for a team of two, three, and four robots in [16].

\section{4 n-Connectivity and TMAP}

For single robots, 1-connectivity in sufficient to solve TMAP. More generally, connectivity of the configuration space plays a vital role in path planning for single robots [8]. However, 1-connectivity is not sufficient to ensure the effectiveness of a team, especially in explorations. Consider the scenario depicted in Figure 5, where node $v$ is an cut point [4] of the navigation course, whose removal results in a disconnected navigation course. The robots, represented by unfilled circles, are located on one side of $v$ and the vertices to be explored (shown in dark circles) are located on the other side. The vertex $v$ must be 
explored first before getting to the other unexplored vertices, and hence acts as a bottleneck.

A graph $G=(V, E)$, for $|V| \geq n+2$ is $n$-connected if it cannot be disconnected by removing $n-1$ vertices [1]. For example, a cycle is 2-connected since removal of any vertex still leaves the rest of the cycle connected. Note that if the graph has a cut point, it is not 2-connected. The following are important consequences of $n$-connectivity:

(a) The degree of every vertex is at least $n$, if not by removing at most $n-1$ neighbors of this vertex, it can be disconnected from the rest of the graph.

(b) If the graph has at least $2 n$ nodes, then for every two disjoint sets $V_{1}$ and $V_{2}$, there exist $n$ disjoint paths joining these two sets of points [4].

The first consequence shows that RVG, VD and DG are not 3-connected. RVG is shown to be 2-connected [17], and we will show that VD and DG are 2 -connected in the next section.

The second consequence is important in robot team exploration as follows. Consider two robots $R_{1}$ and $R_{2}$ located at the vertices of a 2-connected navigation course. There are two vertices $u$ and $v$ to be explored such that they are adjacent to explored part of the graph. Consider that $R_{1}$ has chosen to visit $u$ next. Then $R_{2}$ can get to $v$ without going through $u$. In other words, $R_{2}$ can work around $R_{1}$, when the latter makes the decisions on its own about which vertex to explore next. Assuming that travel time is small, the robots can perform scan operations in parallel, in which case the overall exploration time is halved.

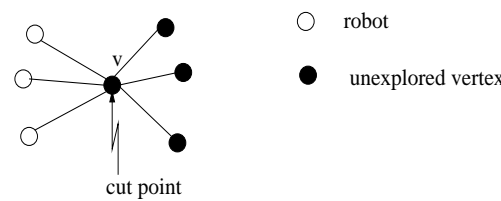

Fig. 5. Illustration of limitations of 1connectivity.

The overall algorithm for a team of $n$ robots is based on the robots executing a graph search algorithm in a cooperative manner using an $n$-connected navigation course. At any step, each robot has the same version of an incomplete navigation course. For the team of robots $R_{1}, R_{2}, \ldots, R_{n}$, let $R_{1}$ have the highest priority, $R_{2}$ have second highest priority, and so on. Each robot performs a scan operation and obtains the resultant visibility polygon. Each robot computes its own adjacency list and communicates it to the other robots. $R_{1}$ sends to $R_{2}$ its next destination $d_{1}$ which is one of the nodes adjacent to its present location. Then $R_{2}$ marks $d_{1}$ as visited and computes its next destination $d_{2}$. $R_{2}$ communicates $d_{1}$ and $d_{2}$ to $R_{3}$, and $d_{2}$ to $R_{1}$. This process is repeated until $R_{n}$ computes its destination. Then the robots move to their chosen destinations and repeat the algorithm. Since the navigation 
course is $n$-connected, all the $n$ robots can get to their respective chosen vertices without being blocked by an unexplored vertex. Informally speaking, $R_{2}$ works around $R_{1}, R_{3}$ works around $R_{2}$ and $R_{1}$, and so on. This algorithm terminates when all known vertices have been visited; the connectivity property together with the terrain-visibility property ensure that the terrain model is completely acquired.

\section{Convex Polygonal Terrains}

In this section, we consider terrains composed of convex polygonal obstacles, and the navigational courses $R V G, V D$ and $D G$.

\subsection{Two-Robot Team}

In the algorithm of previous section, $R_{1}$ is guaranteed to find a destination at each step, due to the connectivity of the navigation course, RVG, VD or DG. We now establish that $R_{2}$ can always find its destination, by showing the 2-connectivity.

RVG is shown to be 2-connected in [16]. For $V D$ and $D G, 2$-connectivity can be shown as follows. The proof method is similar in both cases, and we illustrate the case of VD. For each convex obstacle $O_{1}$, one can trace the cycle of VD that immediately surrounds it. Consider an adjacent obstacle $\mathrm{O}_{2}$ such that it's cycle shares at least an edge with the that of $O_{1}$; then there are three cycles corresponding to each of $O_{i}$ 's and the cycle formed by the union of the two cyles minus the common edges. By extending this argument, it follows that any two vertices of VD belong to some cycle, possibly formed from cycles of different obstacles. Thus, there are two vertex disjoint paths between every pair of vertices, which shows that VD is 2-connected (Theorem $5.10[4])$.

Note that 2-connectivity implies that the navigation course cannot be disconnected by removing a single vertex. Indeed, let the next destination chosen by $R_{1}$ at any step be denoted by $v$. By connectivity, if there is an unvisited node (other than $v$ ), then there is at least one unvisited node adjacent to the paths traced by $R_{1}$ or $R_{2}$. If not, all the unvisited nodes can only be reached via $v$, which makes $v$ a cut point; this in turn contradicts the 2-connectedness. Thus by the time $R_{1}$ performs $\lceil N / 2\rceil$ scan operations, $R_{2}$ would have performed scan operations from the remaining nodes, where $N$ is the number of nodes of the navigation course.

\subsection{Three-Robot Teams}

The Extended Visibility Graph (EVG) is the RVG augmented as follows. The extended hull is obtained by expanding the convex hull of the obstacles by a fixed non-zero amount. Then the vertices of the RVG on the convex hull 
are connected to the corresponding vertices on the extended hull as shown in Fig. 6. The EVG of a terrain cluttered by a finite number of convex polygonal obstacles is shown to be 4-connected and, in general is not 5-connected [16].

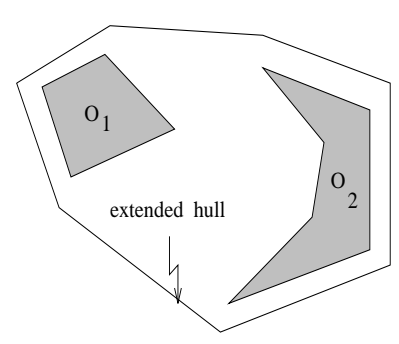

(a) terrain

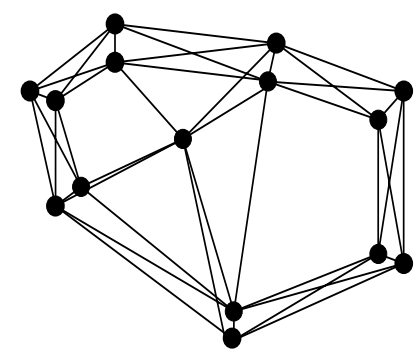

(b) EVG

Fig. 6. Definition of the EVG.

Although, VD and DG are only 2-connected, a 3-robot team can still be effective as shown in Figure 7 for DG. Here $R_{1}$ and $R_{2}$ are forced to stay together at vertices $u$ and $v$, and after that the three robots explore in parallel. In this case $N=11$, and the sensing time is 4 , even though there are only two vertices with degree 3 . A bound on the sensing time can be obtained as follows. Let $N_{2}$ denote the vertices with degree 2 . And $N-N_{2}$ is the number of vertices with degree at least 3 . Nodes with degree 2 can force two robots to stay together, and the other nodes do not. Hence the total sensing time is upper-bounded by

$$
\frac{N-N_{2}}{3}+N_{2} / 2=N / 3+N_{2} / 6 .
$$

Note that for 3 -robot team the best possible sensing time is $N / 3$. If $N_{2}=0$, i.e. the graph is 3 -connected, the bound is $N / 3$. If $N_{2}=N$, i.e. the graph is 2 -connected, the bound is $N / 2$, which is the best possible. In a general case, the effect of the additional term $N_{2} / 6$, which adds to the sensing time, is quite moderate.

\section{General Polygonal Terrains}

For a general polygonal terrain, the navigational course consists of trees which are only 1-connected. The performance of the algorithm for general terrains depends on the number of concave regions and their depths. To tackle this situation, a hierarchical decomposition of the navigation course into $n$-connected and $(n-1)$-or-less-connected components was proposed in [17]; although their main focus is on visibility graph, their decomposition is valid for any navigation course. The performance for the $n(=2,3)$ robot team is expressed in 


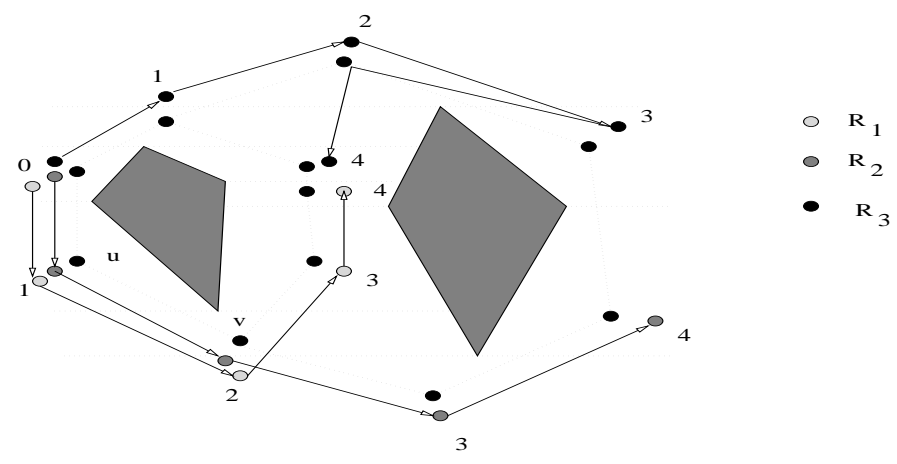

Fig. 7. 3-robot team using DG.

terms of the sizes of $n$-connected components, and the sizes and diameters of $(n-1)$-or-less connected components. This analysis highlights the critical properties such as 2- and 3-connectivity, depth of hierarchy, etc. that support or impede the parallel acquisition of the terrain model.

\section{Conclusions}

The connectivity of the configuration space has been a valuable concept in the terrain model acquisition by single robots. We showed here that the $n$ connectivity plays a similar role for robot teams. The methods based on visibility graphs, Voronoi diagrams and trapezoidal decompositions are shown to yield solutions for efficient terrain model acquisition by a 2- and 3-robot team using visual sensors. This paper is only a first step towards establishing the algorithmic and analytical aspects of mobile robot teams for terrain model acquisition. More generally, it would be of future interest to investigate the role of $n$-connectivity for other applications such as cooperative navigation and search. It would be interesting to see if $n$-connectivity holds for general configurations for non-point robots. Here, only the sensor time is considered here as a measure of performance and the estimates are conservative. We believe that alternative characterizations and better performance estimates are possible. The recently studied class of competitive algorithms for the TMAP by guarantee that the distance traversed by a single robot is bounded by a factor times the minimum possible value achieved if the terrain model is available. Improving the performance of this type of algorithms by employing a team of robots will be of future interest.

\section{Acknowledgements}

This research is sponsored by the Engineering Research Program of the Office of Basic Energy Sciences, U.S. Department of Energy, under Contract No. DE-AC05-96OR22464 with Lockheed Martin Energy Research Corp. 


\section{References}

1. G. Chartrand and L. Lesniak. Graphs and Digraphs. Wadsworth and Brooks/Cole Advaced Books and Software, 1986.

2. H. Choset and J. Burdick. Sensor based motion planning: The hierarchical generalized Voronoi diagram. In Workshop on Algorithmic Foundations of Robotics. 1996.

3. G. Dudek, M. Jenkins, E. Milios, and D. Wilkes. Robust positioning with a multi-agent robotic system. In 1993 IJCAI Workshop Series: Dynamically Interacting Robots, pages 118-123, 1993. Working Notes.

4. F. Harary. Graph Theory. Addison-Wesley Pub., 1969.

5. K. R. Harinarayan and V. J. Lumelsky. Sensor-based motion planning for multiple mobile robots in an uncertain environment. In Proceedings of IEEE/RSJ/GI Int. Conf. on Intelligent Robots and Systems, pages 1485-1492, 1994.

6. K. Ishioka, K. Hiraki, and Y. Anzai. Cooperative map generation by heterogeneous autonomous mobile robots. In 1993 IJCAI Workshop Series: Dynamically Interacting Robots, pages 57-67, 1993. Working Notes.

7. Y. Ishiwata, M. Inaba, and H. Inoue. Cooperative recognition of environments by multiple robots. In Proc. of JSME Annual Conf. on Robotics and Mechatronics, pages 79-84, 1992

8. J. C. Latombe. Robot Motion Planning. Kluwer Academic Pub., Boston, 1991.

9. V. Lumelsky, S. Mukhopadhyay, and K. Sun. Dynamic path planning in sensorbased terrain acquisition. IEEE Transactions on Robotics and Automation, 6(4):462-472, 1990.

10. N. S. V. Rao. Algorithmic framework for learned robot navigation in unknown terrains. IEEE Computer, 22:37-43, 1989.

11. N. S. V. Rao. On fast planning og suboptimal paths amidst polygonal obstacles in plane. Theoretical Computer Science, 140:265-289, 1995.

12. N. S. V. Rao. Robot navigation in unknown generalized polygonal terrains using vision sensors. IEEE Transactions on Systems, Man and Cybernetics, 25(6):947-962, 1995 .

13. N. S. V. Rao and S. S. Iyengar. Autonomous robot navigation in unknown terrains: Visibility graph based methods. IEEE Transactions on Systems, Man and Cybernetics, 20(6):1443-1449, 1990.

14. N. S. V. Rao, S. S. Iyengar, B. J. Oomen, and R. L. Kashyap. On terrain model acquisition by a point robot and polyhedral obstacles. IEEE Journal of Robotics and Automation, 4:450-455, 1988.

15. N. S. V. Rao, S. Kareti, W. Shi, and S. S. Iyengar. Robot navigation in unknown terrains: An introductory survey of non-heuristic algorithms. Technical Report ORNL/TM-12410, Oak Ridge National Laboratory, Oak Ridge, TN, 1993.

16. N. S. V. Rao, N. Manickam, and V. Protopopescu. Connectivity of visibility graphs and terrain model acquisition by robot teams. Congressus Numerantium, 120:129-137, 1996.

17. N. S. V. Rao, N. Manickam, and V. Protopopescu. Cooperative terrain model acquisition by a team of two or three point-robots. In Proc. Int. Conf. on Robotics and Automation, pages 1427-1433, 1996.

18. N. S. V. Rao, N. Stoltzfus, and S. S. Iyengar. A 'retraction' method for learned navigation in unknown terrains. IEEE Transactions on Robotics and Automation, 7(5):699-707, 1991. 